\title{
Gender Differences in Frontal Activation while Perceiving Pathologically Thin Female Body Forms
}

\author{
Toru Uehara1, Yoko Ishige ${ }^{2}$, Masashi Suda ${ }^{2}$, Perminder Sachdev ${ }^{3,4}$ \\ ${ }^{1}$ General Health Support Center, Gunma University, Maebashi, Japan \\ ${ }^{2}$ Department of Neuropsychiatry, Gunma University Graduate School of Medicine, Maebashi, Japan \\ ${ }^{3}$ School of Psychiatry, University of New South Wales, Sydney, Australia \\ ${ }^{4}$ Neuropsychiatric Institute, Prince of Wales Hospital, Sydney, Australia \\ E-mail: toruaki@gunma-u.ac.jp \\ Received March 23, 2011; revised May 13, 2011; accepted June 18, 2011
}

\begin{abstract}
Brain mechanisms underlying body image disturbances are a focus of research in the realm of eating disorders, and functional imaging studies have revealed gender differences in the processing of body shape. In this study, using 16-channel near infrared spectroscopy, we investigated frontal lobe activation in 46 healthy university students during viewing of photographs of pathologically thin female forms, and compared gender differences in activation, and examined the correlations between the relative changes in cerebral blood volume, eating attitudes, and perceived feelings. Participants completed the Eating Attitudes Test (EAT26) and rated a visual analogue scale for anxiety/disgust. Significant gender differences in the pattern of activation were noted in the prefrontal region (predominantly right side, dorsolateral to ventral), with male participants showing greater and more widespread frontal activation. The total and subscale scores on EAT26 were significantly correlated with the frontal activation, and perceived feelings were significantly associated with increased prefrontal activation on the left side. Gender differences in frontal activation suggest differential expectations between men and women of pathologically thin female body forms. The study results suggest that anorexic psychopathology may be associated with abnormal right frontal activation while viewing thin bodies of others.
\end{abstract}

Keywords: Body Image, Anorexia Nervosa, Prefrontal Cortex, Eating Attitudes, Near Infrared Spectroscopy

\section{Introduction}

The brain mechanisms underlying body image processing have been explored in previous studies, using a variety of methodologies. An investigation of the neural processing of body shape revealed activation in a distributed network, including the lateral fusiform gyrus, lateral prefrontal cortex (PFC), and right parietal cortex [1]. The dorsolateral PFC and the insular, inferior parietal, fusiform, and anterior cingulate (AC) cortical areas have been demonstrated to respond to images of one's own distorted body [2]. Other studies have shown that lesions in the dorsolateral PFC and/or parietal cortex were associated with impaired performances on tasks requiring on-line coding of the body posture [3]. Derogatory words concerning one's own body image have been reported to provoke activations in the temporomesial area, including the amygdala, in young women [4].
In terms of clinical correlations, a recent fMRI study [5] reported that anorexia nervosa (AN) patients demonstrated stronger activation of the insula and lateral PFC cortex during the satisfaction rating of thin self-images, and the study indicated a stronger emotional involvement while the subjects were presented with distorted images close to their own ideal body size. Friederich et al. [6] summarized that a neural network could be involved in the general processing of body images (the lateral fusiform, parietal and dorsolateral PFC) and a related 'emotional' network (AC, insula, amygdala) may be activated when body-shape-related stimuli induce self-related or emotional reactions. Consequently, these neural connections might play a significant role in the pathophysiology of eating disorders, and possibly in the gender-related difference in the prevalence of eating disorders.

However, several complex processes could be involved in the brain response, as seen in the detected activations, 
according to the content or focus of the stimuli. For example, activation of the left ventrolateral and apical PFC was found in a previous fMRI study in which line drawings of underweight body shapes were used [1]; on the other hand, exposure to distorted images of one's own body showed activations of the left [7] or bilateral [2] ventrolateral PFC. On the other hand, the dorsal division of the right AC (BA 32) has been suggested to be important for self-referential processing of emotions $[8,9]$. In anxiety responses to pictures of slim body shapes, Friederich et al. [6] commented that differential activation of the fear network, including the amygdala and the AC, was associated with substantial body dissatisfaction. Based on these studies, it seems reasonable to divide task profiles into individual factors, including pure self-reference and objective thin stimulation. In response to the respective paradigms, one could evaluate subjective and objective feelings or reactions while presenting visual stimuli.

We assume that body image processing can be divided into several components according to the cognitive processes involved; first, simple self-monitoring while comparing with others; second objective stimulation by body forms (e.g., emaciation or obesity); and last, disturbance of body image perception, such as drawings of body shapes or distorted graphics. Therefore, we conducted the present study with the objective of selecting one component of these processes, i.e. "how do young adults feel about or process others' pathological emaciation?” We used stimuli that have been previously used successfully (body forms of female patients with AN) [10]. This aforementioned study indicated that processing of non self-images by control subjects activated the inferior and middle frontal gyri, and the superior and inferior parietal lobules. AN patients had a similar pattern of activation with greater activation of the medial frontal gyrus. Interestingly, when the differential activation in the 2 groups was investigated with self vs. non-self-images, control subjects showed greater activation in the middle frontal gyri and insula than the patients, while the patients did not show greater activation in any region. Sachdev et al. [10] concluded that discrepant emotional and perceptual processing may underlie the distortion of self-images by AN patients. It is very important to determine whether Sachdev et al.'s study findings apply equally to men and women from a different cultural background.

We used near infrared spectroscopy (NIRS) for this study because of its noninvasiveness and ease of performance. NIRS employs near-infrared light emitted and detected on the skull skin [11]. It allows the monitoring of hemodynamic changes, including both cerebral blood volume changes and the oxygenation state, using a small apparatus with a high time resolution of about 0.1 sec- onds. It also allows the monitoring of changes in the oxygenated hemoglobin concentration (o- $\mathrm{Hb})$ and deoxygenated hemoglobin concentration (d-Hb). NIRS is considered to be suitable for studies of higher brain functions because it enables measurements in the natural setting as compared with other brain imaging techniques: for example, subjects can undergo NIRS examination in the sitting position, with their eyes open, or while speaking and sitting on a bed [12]. Taking advantage of these characteristics, several NIRS studies have been conducted in patients with psychiatric disorders, such as schizophrenia [13,14], depression [15], and attentiondeficit/hyperactivity disorder [16]. These characteristics have also enabled the investigation of subjective, delicate experiences in healthy subjects, such as subjective sleepiness and conversations $[17,18]$.

\section{Methods}

\subsection{Participants}

The study participants were 46 healthy university students (32 females), with a mean age of 20.5 years (S.D. 2.2). Two were left-handed, and 3 were visiting students from China, the others being Japanese. Their average body weight and height were $55( \pm 7.0) \mathrm{kg}$ and $162( \pm$ 8.9) $\mathrm{cm}$, respectively, and the body mass index (BMI) ranged from 18 to 24 (mean 21.3 for all; 22.5 for male and 20.8 for female). None of the participants had any significant medical/psychiatric history. They were voluntarily recruited as subjects of this scientific study, and were paid 1600 yen, according the official provision, for their co-operation in the exam. All subjects gave written informed consent prior to their participation in the study, in conformity with the provisions of the Declaration of Helsinki, revised in Edinburgh in 2000. We strictly protected the privacy of the subjects, carefully preserving their anonymity. The data collection was conducted from August to December in 2009 and from August to December in 2010.

\subsection{NIRS Machine}

NIRS allows calculation of the changes in the hemoglobin parameters, including oxygenated hemoglobin [o-Hb] and deoxygenated hemoglobin [d-Hb], by measuring attenuation of the near-infrared light around the wavelength of $800 \mathrm{~nm}$. The neural activation induces regional cerebral blood dynamics. It induces hemodynamic changes in the brain tissue, almost identical in pattern to spontaneous cerebral neural activity. Cortical activation is typically detected as an [o-Hb] increase and [d-Hb] decrease; however, the direction of changes of $[\mathrm{d}-\mathrm{Hb}]$ 
can be ambiguous in the frontal lobe [19]. Mainly, changes of [o-Hb] at a depth of $2-3 \mathrm{~cm}$ from the scalp, that is, the surface of the cerebral cortex, are considered to be correlated with the hemodynamic changes in PET [20] and blood oxygenation level-dependent (BOLD) signal changes in fMRI [21,22]. NIRS does not measure cerebral luminescence, but measures attenuation of the irradiated light intensity. Therefore, the combination of optical irradiation and photon detection determines the resolution. NIRS characteristically measures not the 1:1 combination of irradiation and detection, but the light from one light source with 2 or more detectors arranged geometrically in the NIRS measurement system. Thus, information on which detector measures the signal of which portion becomes important. There are a number of methods for judging this channel separation. The first method, Time Division Multiple Access (TDMA), makes a light source turn on in turns and separates a signal on a time-axis. The second method, Frequency Division Multiple Access (FDMA), is for modulating and irradiating 2 or more light sources with different frequencies and separating a signal based on the frequency information after detection. The third method is Code Division Multiple Access (CDMA) which uses spectrum diffusion attenuation and is applied GPS or mobile phone technology. The NIRS machine we used, OEG-16 (Spectratech, Inc, Japan), employs CDMA methodology and is, therefore, very convenient and portable. It can provide NIRS data under natural conditions non-invasively, and artifacts induced by hair can be avoided because of the adjustments which allow reading only of the front of the head. The OEG-16 measures 16 channels on the frontal lobe (according to Broadman's map, it provides data approximately on sections $10,11,12,44,45$, and 46). Its time resolution is 0.5 seconds and space resolution is 2 $\mathrm{cm}$. The headset was placed according to the $10 / 20$ system, by which a central hole was coordinated with Fz. From channels 1 to 8 , the measurement points were placed from the right to the central pole. From channels 9 to 16 , the measurement points were placed from the ventral/rostral to the left lateral (to refer the video content). It provides relative changes of hemoglobin concentration and values are obtained in arbitrary units (concentration $\times$ path length).

\subsection{Task}

A visual paradigm was used in which 3-dimensional (front, side, and back) photos of 2 pathologically thin women (BMI are ranged from 15-16), used in a previous fMRI study, were presented [10]. The women were dressed in standardized clothing (white cropped singlet with the abdomen showing, black bike pants and no jew- ellery), so as to reveal the body contour. The faces of all the images were digitally masked to avoid distraction from the body form. The body images were placed against a uniform grey background of medium-intensity. Digital images were prepared using Photoshop ${ }^{\circledR}$. To maintain the subjects' attention during the viewing, the subjects were instructed to express how he/she thinks or feels about comparing the images with him/herself. The different body forms were shown in 3 different profiles for an equal duration, i.e., front, back, and side, switched every 5 seconds. The baseline image was a uniformly grey screen of medium intensity which was identical to the background of the body images (pre-task 5 sec, intertask 5 sec, and post-task $5 \mathrm{sec}$ ).

\subsection{Perceived Feeling and EAT}

Perceived feeling about thinner images was rated on a scale of 0 - 10, according to the participants' overall impression. The anxiety (how have you felt anxiety?) and disgust (how have you felt disgust?) levels were scaled on a visual analogue scale soon after viewing pictures subjectively.

Eating attitudes were assessed in all of the subjects using the Japanese version of the Eating Attitude Test (EAT-26) $[23,24]$ at the time of the examination. EAT-26 is a 26-item self-rated questionnaire for evaluating eating problems. The results are presented as the total score (range, 0-78), as well as the scores on the 3 subscales, namely, diet (range, 0 - 39), bulimia and food preoccupation (range, 0 - 18), and oral control (range, 0 - 21), which respectively reflect avoidance of fattening foods and preoccupation with being thinner, thoughts about food as well as items indicating bulimia, and self-control in relation to eating and perceived pressure from others to gain weight.

\subsection{Data Analysis}

The continuous waveforms of [Hb] changes in all of the 16 channels were acquired from each of the subjects during the paradigm. The individually averaged [Hb] waveforms were obtained as the average sum of 2 trials: the baseline realignment for 5 sec before and after the task periods, and a task segment averaging 2 sets of 15sec image viewing periods. Thereafter, the grand average values of the baseline and task segments in each channel were calculated for all of the participants' data. We used only [o-Hb] values as cerebral blood volume changes were based on a previous report $[25,26]$. Topography (video content) was presented according to the time course on the frontal portion. For this grand averaged data, channels which carried significant activations were ana- 
lyzed using the t-test between the pre-task and task periods (http://www.brsystems.jp). Differences in the mean values were tested by combined variance, as the number of samples for the 2 periods was not equal.

Combined variance: Ue2 $=\{($ na -1$)$ ua $2+$

$$
(n b-1) u b 2\} /\{n a+n b-2\}
$$

Variance and numbers of sample a: ua2 and na

Variance and numbers of sample b: ub2 and nb

T-value: $\mathrm{t} 0=|\mathrm{mXa}-\mathrm{mXb}| / \operatorname{root}\{$ ue2(1/na $+1 / \mathrm{nb})\}$

Average of each sample: $\mathrm{mXa}, \mathrm{mXb}$

In the next step, gender differences were investigated using a $t$-test for each channel during the task period by analyzing the differences in the grand average data between males and females. In the last step, the relationships of the [o-Hb] changes with the perceived anxiety/ disgust and EAT scores (total and subscales) were investigated. Channels with significantly correlated changes (diet, bulimia and food preoccupation, and oral control scores for EAT-26, BMI, VAS score) were analyzed by non-parametric correlation. An imaging software was used to analyze NIRS parameters (Data Viewer ver.1.1a, BR Systems. Inc., Japan). The other statistical analyses were conducted using SPSS version 17.0 (SPSS Japan, Inc.).

\section{Results}

\subsection{Waveform and Gender}

The Figure 1 demonstrates the grand average waveforms for the $[\mathrm{Hb}]$ changes for all participants according to gender (for males on the left side). The red polygonal line indicates the relative changes of [o-Hb], the blue indicates those of $[\mathrm{d}-\mathrm{Hb}]$, and the green indicates the changes of the total- $\mathrm{Hb}$ (sum of o- and d-Hb). Overall, fluctuating alterations were generally seen in many channels during the task. The video content depicts the topography of the [o-HB] changes (for females on the right side), and redder areas represent higher activations (grading to yellow, green, and opposite in deeper blue). $<$ Video content can be viewed on http://www.youtube. $\mathrm{com} /$ watch?v=Z24uVzzEZO4>

Comparisons between the baseline and task periods are ummarized in Table $\mathbf{1}$ according to gender. The respective $t$-value for each channel showed significant activations $(p<0.05$, DoF $=24)$ on channels $1,2,3$, and 11 (dominantly right lateral PFC) in males, and significant activations $(p<0.05, \mathrm{DoF}=24)$ on channel 5 and 8 (right PFC) and significant deactivations in channel 6 (right lower ventral PFC) in females. Comparisons of activations for the task periods between the males and females are presented in Table 2; $t$-values for every
Table 1. Comparisons of oxy-hemoglobin changes between rest and task periods.

\begin{tabular}{ccccccc}
\hline & \multicolumn{3}{c}{ male } & & \multicolumn{3}{c}{ female } \\
\hline & rest & task & $t$ & rest & task & $t$ \\
\hline sample & 4 & 22 & & 4 & 22 \\
channel 1 & -0.003 & 0.043 & $-2.78^{*}$ & 0.002 & 0.000 & 0.41 \\
2 & -0.006 & 0.021 & $-2.11^{*}$ & 0.004 & -0.004 & 1.99 \\
3 & 0.002 & 0.022 & $-2.48^{*}$ & -0.001 & -0.001 & 1.01 \\
4 & 0.005 & 0.037 & -1.96 & -0.001 & 0.000 & -1.35 \\
5 & 0.000 & 0.004 & -1.10 & -0.001 & 0.003 & $-3.52^{*}$ \\
6 & 0.003 & 0.020 & -1.80 & -0.001 & -0.004 & $2.30^{*}$ \\
7 & 0.000 & 0.011 & -1.48 & -0.001 & 0.000 & -0.63 \\
8 & -0.003 & 0.020 & -1.58 & -0.002 & 0.003 & $-4.56^{*}$ \\
9 & -0.003 & -0.014 & 0.52 & -0.001 & -0.001 & -0.06 \\
10 & 0.005 & 0.004 & 0.09 & 0.000 & -0.002 & 1.51 \\
11 & 0.002 & 0.021 & -2.01 & 0.000 & 0.000 & 0.54 \\
12 & 0.002 & 0.006 & -0.52 & -0.001 & -0.001 & 0.07 \\
13 & 0.004 & 0.029 & -1.87 & 0.002 & -0.001 & 0.78 \\
14 & 0.009 & 0.025 & -1.14 & 0.001 & 0.001 & 0.24 \\
15 & 0.004 & -0.005 & 1.00 & 0.004 & 0.001 & 1.15 \\
16 & 0.008 & -0.027 & 1.64 & 0.009 & 0.006 & 0.41 \\
\hline
\end{tabular}

Degree of freedom $=24, * ; p<0.05$, rest vs. task.

Table 2. Comparisons of activations in task periods between male and female.

\begin{tabular}{|c|c|c|c|c|}
\hline channel & male & female & $t$ & $p$ \\
\hline 1 & 0.045 & 0.000 & 7.47 & $* *$ \\
\hline 2 & 0.022 & -0.004 & 5.44 & $* *$ \\
\hline 3 & 0.024 & -0.001 & 9.31 & $* *$ \\
\hline 4 & 0.039 & 0.000 & 6.82 & $* *$ \\
\hline 5 & 0.005 & 0.003 & 1.64 & \\
\hline 6 & 0.022 & -0.004 & 8.26 & $* *$ \\
\hline 7 & 0.012 & 0.000 & 4.61 & $* *$ \\
\hline 8 & 0.020 & 0.003 & 3.21 & $*$ \\
\hline 9 & -0.014 & -0.001 & -1.66 & \\
\hline 10 & 0.005 & -0.002 & 1.50 & \\
\hline 11 & 0.023 & 0.000 & 8.08 & $* *$ \\
\hline 12 & 0.007 & 0.000 & 3.09 & * \\
\hline 13 & 0.032 & 0.000 & 8.03 & $* *$ \\
\hline 14 & 0.028 & 0.000 & 5.18 & $* *$ \\
\hline 15 & -0.003 & 0.002 & -1.56 & \\
\hline 16 & -0.029 & 0.006 & -4.13 & $* *$ \\
\hline
\end{tabular}

Degree of freedom $=24, * ; p<0.05$, rest vs. task. 

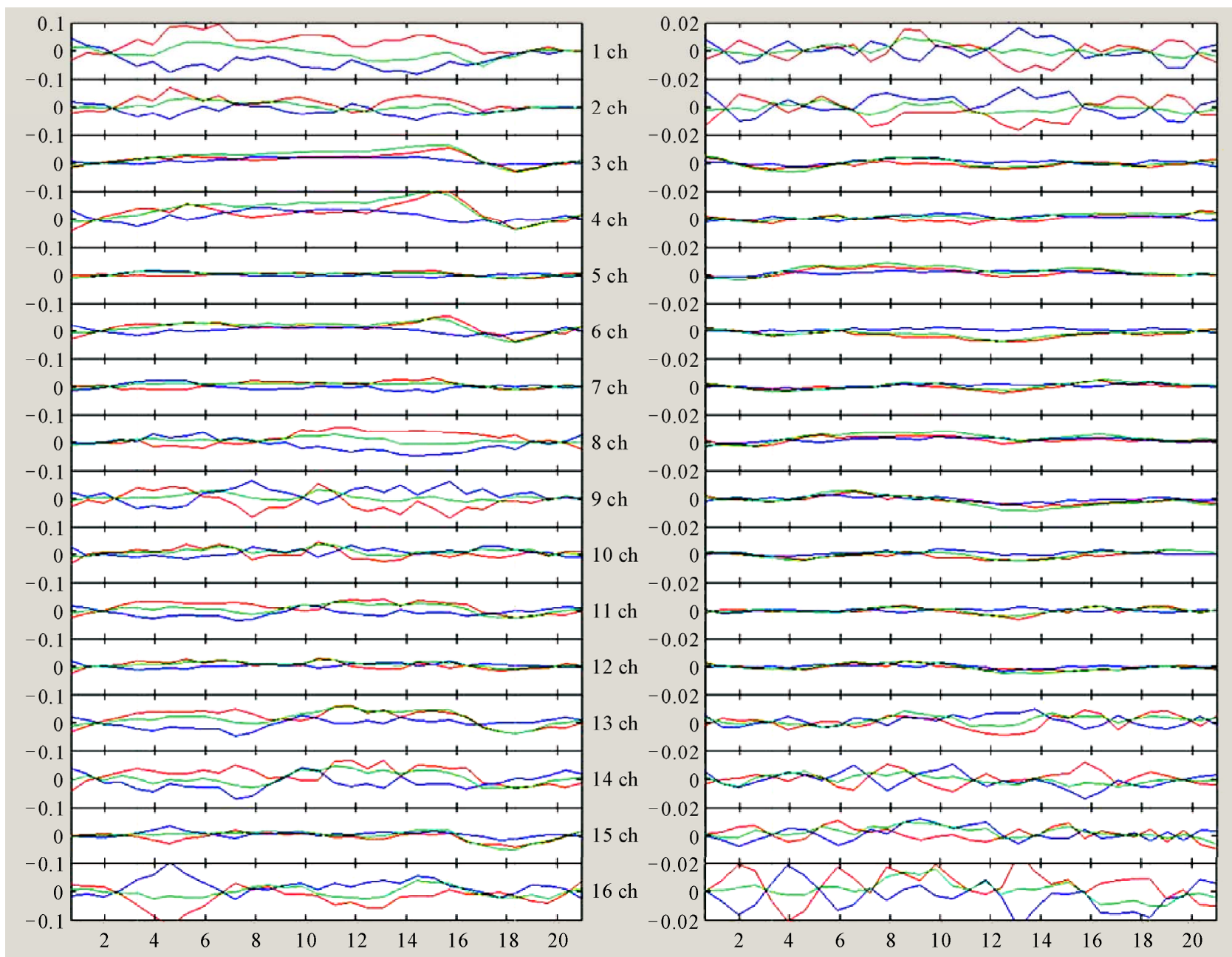

Figure 1. This figure demonstrates the grand average waveforms for the [Hb] changes in all of the participants according to gender (for males on the left side). The red polygonal line indicates the relative changes of the [o-Hb], the blue polygonal line indicates those of [d-Hb], and the green polygonal line indicates the total [Hb] (sum of o-HB and d-HB) relative changes. The vertical axis shows the relative changes of $[\mathrm{Hb}](\mathrm{mMmm})$, and the vertical grey lines show the mean start and end of the task periods. The numbers on the axis of abscissas indicate the time-point of the measurements (seconds). The number besides each waveform indicates respective channel as follows; from channels 1 to 8, the measurement points were placed from the right to the central pole. From channels 9 to 16, the measurement points were placed from the ventral/rostral to the left lateral (to refer the video content).

channel and significant differences are presented. Overall, males showed more activations than females in frontal functioning, with especially significant differences ( $p<$ 0.01, DoF $=23.4$ ) on channels 1 - 4, 7 - 8 (right PFC), 11, 13 - 14 (left PFC). In contrast, channel 16 (left lateral) showed significantly greater activations in females than in males $(p<0.01)$.

\subsection{Correlation with EAT and Perceived Feelings}

Spearman's correlation coefficients were calculated for the relative changes of [o-Hb] and the scores on EAT26 or perceived feelings (rho $>0.30,{ }^{*} p<0.05$; rho $>0.41$, $\left.{ }^{* *} p<0.01\right)$. Table 3 shows the correlation coefficients on the respective channels. The total EAT 26 scores were correlated with activations of channels $1-4^{* *}, 6^{* *}, 7^{*}$ (right lateral to ventral PFC), 12*, and 15* (left lateral PFC). The OC subscale scores were correlated with activations of channels $1^{*}, 2^{* *}, 3-4^{*}, 6^{* *}, 7^{*}$ (right lateral to medial PFC), and the diet subscale scores were correlated with activations of channels $1^{*}, 2^{* *}, 4^{*}$, and $6^{*}$ (right PFC). The BE subscale scores were correlated with activations of channels $1-2 *, 3-4 * *, 5 *, 7 *$ (right PFC) and $12^{*}, 15^{* *}, 16^{*}$ (left lateral PFC). The scores for perceived disgust were correlated with activations of the left prefrontal channels $\left(11^{* *}, 13^{* *}\right.$, and $\left.14^{*}\right)$, and those for perceived anxiety were correlated with activations of 
Table 3. Correlations between perceived feelings or EAT and NIRS activations.

\begin{tabular}{|c|c|c|c|c|c|c|c|c|c|c|c|c|c|c|c|c|c|}
\hline & & ch1 & ch2 & ch3 & ch4 & ch5 & ch6 & ch7 & ch8 & ch9 & $\operatorname{ch} 10$ & ch11 & ch12 & ch13 & ch14 & ch15 & $\operatorname{ch} 16$ \\
\hline \multirow{2}{*}{ anxiety } & $r$ & 0.08 & -0.03 & 0.15 & 0.16 & 0.06 & 0.13 & 0.20 & 0.11 & 0.08 & 0.02 & 0.26 & 0.02 & $0.317^{*}$ & 0.23 & 0.09 & 0.04 \\
\hline & $p$ & 0.60 & 0.82 & 0.32 & 0.30 & 0.70 & 0.41 & 0.19 & 0.47 & 0.62 & 0.90 & 0.08 & 0.87 & 0.03 & 0.12 & 0.57 & 0.77 \\
\hline \multirow{2}{*}{ disdust } & $r$ & 0.25 & 0.18 & 0.17 & 0.24 & 0.06 & 0.16 & 0.23 & 0.00 & 0.15 & 0.03 & $0.403^{* *}$ & 0.11 & $0.421^{* *}$ & $0.321^{*}$ & -0.07 & -0.10 \\
\hline & $p$ & 0.10 & 0.25 & 0.26 & 0.11 & 0.71 & 0.31 & 0.13 & 0.99 & 0.32 & 0.85 & 0.01 & 0.46 & 0.00 & 0.03 & 0.64 & 0.50 \\
\hline \multirow{2}{*}{ EAT total } & $r$ & $0.487^{* *}$ & $0.632^{* *}$ & $0.409^{* *}$ & $0.422^{* *}$ & 0.29 & $0.461^{* *}$ & $0.319^{*}$ & 0.17 & 0.13 & 0.20 & 0.28 & $0.376^{*}$ & 0.22 & 0.21 & $0.354^{*}$ & 0.16 \\
\hline & $p$ & 0.00 & 0.00 & 0.01 & 0.00 & 0.05 & 0.00 & 0.03 & 0.25 & 0.39 & 0.18 & 0.06 & 0.01 & 0.14 & 0.17 & 0.02 & 0.28 \\
\hline \multirow{2}{*}{ diet } & $r$ & $0.348^{*}$ & $0.424^{* *}$ & 0.23 & $0.313^{*}$ & 0.10 & $0.297^{*}$ & 0.11 & 0.11 & 0.07 & 0.15 & 0.23 & 0.25 & 0.13 & 0.19 & 0.17 & 0.03 \\
\hline & $p$ & 0.02 & 0.00 & 0.13 & 0.04 & 0.51 & 0.05 & 0.46 & 0.49 & 0.64 & 0.32 & 0.12 & 0.10 & 0.38 & 0.20 & 0.26 & 0.87 \\
\hline \multirow{2}{*}{$\begin{array}{c}\text { oral } \\
\text { control }\end{array}$} & $r$ & $0.324^{*}$ & $0.462^{* *}$ & $0.315^{*}$ & 0.28 & 0.23 & $0.425^{* *}$ & $0.295^{*}$ & -0.01 & -0.01 & 0.05 & 0.23 & 0.29 & 0.18 & 0.21 & 0.23 & 0.14 \\
\hline & $p$ & 0.03 & 0.00 & 0.04 & 0.06 & 0.13 & 0.00 & 0.05 & 0.93 & 0.95 & 0.75 & 0.13 & 0.05 & 0.25 & 0.17 & 0.13 & 0.37 \\
\hline \multirow{2}{*}{$\begin{array}{l}\text { binge } \\
\text { eating }\end{array}$} & $r$ & $0.313^{*}$ & $0.342^{*}$ & $0.429^{* *}$ & $0.381^{* *}$ & $0.365^{*}$ & 0.28 & $0.367^{*}$ & 0.29 & 0.16 & 0.17 & 0.14 & $0.344^{*}$ & 0.21 & 0.09 & $0.439^{* *}$ & $0.313^{*}$ \\
\hline & $p$ & 0.04 & 0.02 & 0.00 & 0.01 & 0.01 & 0.06 & 0.01 & 0.06 & 0.30 & 0.27 & 0.37 & 0.02 & 0.17 & 0.57 & 0.00 & 0.04 \\
\hline \multirow{2}{*}{$\begin{array}{l}\text { body mass } \\
\text { index }\end{array}$} & $r$ & 0.07 & 0.03 & -0.19 & -0.14 & -0.12 & -0.13 & -0.20 & -0.03 & 0.02 & -0.09 & -0.21 & -0.11 & -0.35 & -0.14 & $-0.367^{*}$ & -0.33 \\
\hline & $p$ & 0.70 & 0.89 & 0.30 & 0.48 & 0.52 & 0.51 & 0.30 & 0.85 & 0.93 & 0.64 & 0.27 & 0.58 & 0.06 & 0.47 & 0.05 & 0.08 \\
\hline
\end{tabular}

Spearman's correlation coefficients, ${ }^{* *} ; p<0.05, * ; p<0.05$ ch: channel.

channel 13*.

\section{Discussion}

The present study attempted to estimate the prefrontal activations induced by visual stimuli consisting of the body forms of anorexia patients, using noninvasive and simple-to-perform functional brain imaging. Importantly, the subjects were asked to compare the abnormally thin forms presented with their own body image, thereby making the presentations emotionally relevant. This visual task was associated with widespread and fluctuating activations, but predominantly of the right PFC. This finding was in agreement with the results of previous studies, for instance, the fMRI study by Sachdev and colleagues [10] which indicated that non-self-images induced activations in the medial inferior frontal gyrus (BA46), and the fMRI study in which females without eating disorder (ED) presented with pictures of slim models showed activations in the right PFC and the inferior frontal pole (BA9) [6].

Interestingly, obvious gender differences were observed in the widespread PFC activations, and male students showed significantly stronger activations bilaterally in the PFCs than females. There are a number of possible explanations for this gender difference. If bilateral activation of the PFC indicates more focused attention and active processing of the images, males seem to have been more actively engaged with these images. On the other hand, women were either not so engaged or in fact actively suppressed the processing of the images which they were visualizing. Female participants showed deactivations in the right lower ventral PFC, which may indicated active suppression during body image processing in females. In fact, the rostral PFC is involved in the processing of conflicting and salient stimuli and top-down inhibition of amygdala activity [27]. It might be interpreted as suggesting that deactivation of the right PFC (maybe rostral) in healthy young women reflects the sensitivity to negative body forms by self-comparison. Another possible explanation is that the body image of women has changed toward thinness to such an extent that they did not find the stimuli too discordant from their own bodies, and thereby showed low interest and activation. Men, on the other hand, still found these female forms to be pathologically thin.

The other interesting findings from the present study were specific correlations between the PFC activations and the scores on EAT and for perceived feelings. In students with higher diet or oral control scores, presentation of thinner images produced activations in the right PFC. On the other hand, students with higher food preoccupation scores showed bilateral activations along the left PFC. In contrast, students with higher perceived feelings (disgust or anxiety) when presented with thinner others' images showed only left PFC activations. These findings suggest specific links between the respective eating patterns and the laterality of PFC activation. In 
our related study on eating disorders [28], we found functional relationships between eating psychopathology and brain activations and concluded that the clinical symptoms of ED consisted of 2 components: dieting tendency, correlated with the right frontotemporal cortex, and eating behavior problems (including binge eating) correlated with the left orbitofrontal cortex. Linkage of left PFC activation to fear of fatness or desire for thinness, and linkage of right PFC activation to bulimia might also be a continuum in healthy subjects. The existence of relationships between body images, eating attitudes, emotion and PFC activations might be speculated to indicate specific ED-related neural mechanisms. In addition, Friederich et al. [6] commented that individual differences in anxiety ratings in response to presentation of body shape models were positively associated with activations of the fear network, including in the rostral PFC and the inferior lateral PFC. According to that author, activation of the ventrolateral PFC (BA 47) is involved in anxiety processing [29] and anger [30]. Another study suggests that ventrolateral PFC activation reflects the intensity dimension of emotional perception rather than the processing of discrete emotions [31]. The present study also found that females showed significantly higher activations only in the left lateral PFC (channel 16) as compared to males, and this may be related to gender-specific emotional status and processing.

Some limitations of this study should be noted. We used NIRS for studying brain activation, unlike previous studies that have mostly used BOLD fMRI. When applying NIRS to the clinical setting, relative changes of cerebral hemoglobin data should be cautiously interpreted as these are not absolute values. Also, anatomical identification of the measurement points is one of the major methodological challenges in multichannel NIRS; however, a virtual registration method has been developed recently to improve placement and detection [32]. The spatial resolution in NIRS ranges from 2 to $3 \mathrm{~cm}$; therefore, it is possible to discuss functional dynamism in the context of broader frontal connectivity. NIRS does offer high time resolution, which can contribute to clarification of the networking or circuit by allowing analyses of the time-course and pattern of activations. NIRS, as used in our study, is limited to the examination of the frontal lobes and we therefore cannot comment on other brain regions involved in these processes, which is another limitation of our work. The study of body image processing can benefit from many paradigms. Most previous studies have focused on self-images, distorted thin images, or estimating body weights. It may be reasonably expected that the extrastriate body area, which is located in the lateral occipitotemporal cortex, must respond to visual images of human bodies and body parts
[33]. In fact, one study suggested the importance of the right parietal cortex in developing and maintaining body representation, as well as in the pathogenesis of anorexia [34]. This study used a frontal-specific methodology, and should therefore be complemented with whole-brain measurement. Considering the importance of body image disturbance, especially for the mental health of women, and the interest in the neurobiological basis of gender differences in EDs, the clinical implications of these findings should be investigated. Recently, Miyake et al. [35] reported significant activation of the occipitotemporal cortex, right parietal cortex, and DLPFC (BA 9) in restricting AN using the other fat-image task versus the other real-image task. The occipitotemporal and right parietal cortices were significantly activated in the binge eating/purging type of AN (AN-BP) and control subjects, whereas in the bulimia nervosa (BN) patients, the right occipital (BA 18) and right parietal lobes were significantly activated. Their series of studies used negative word stimuli concerning body, and the left medial PFC was activated both in patients with BN and in patients with AN-BP [36].

The other limitations of this study should be noticed. Female participants were over two folds than male participants in this study; therefore, it must be careful to generalize this finding. And people in different cultures may have different response to pathologically thin female body forms. Actually regarding psychopathology of eating disorders, thinner body image could be common within various Westernized countries. But it should be mentioned that gender difference may not be observed for people in alternative areas.

In conclusion, gender differences in frontal activation suggest differential processing by men and women of pathologically thin female body forms. The study also suggests that anorexic psychopathology may be associated with abnormal right frontal activations while viewing the thin body forms of others.

\section{Acknowledgements}

This study was supported by the Intramural Research Grant (20-1) for Neurological and Psychiatric Disorders of NCNP, Japan. The summary of this study have been presented at a national meeting (33rd Japanese Society of Biological Psychiatry in Tokyo, 2011) and will be on an international conference (17th Eating Disorders Research Society in Edinburgh, 2011).

\section{References}

[1] R. Uher, T. Murphy, H. C. Friederich, T. Dalgleish, M. J. Brammer, V. Giampietro, M. L. Phillips, C. M. Andrew, 
V. W. Ng, S. C. Williams, I. C. Campbell and J. Treasure, "Functional Neuroanatomy of Body Shape Perception In Healthy and Eating-Disordered Women," Biological Psychiatry, Vol. 58, No. 12, 2005, pp. 990-997. doi:10.1016/j.biopsych.2005.06.001

[2] A. Wagner, M. Ruf, D. F. Braus and M. H. Schmidt, "Neuronal Activity Changes and Body Image Distortion in Anorexia Nervosa," NeuroReport, Vol. 14, 2003, pp. 2193-2197. doi:10.1097/00001756-200312020-00012

[3] J. Schwoebel and H. B. Coslett, "Evidence for Multiple, Distinct Representations of the Human Body," Journal of Cognitive Neuroscience, Vol. 17, No. 4, 2005, pp. 543553. doi:10.1162/0898929053467587

[4] N. Shirao, Y. Okamoto, T. Mantani, Y. Okamoto and S. Yamawaki, "Gender Differences in Brain Activity Generated by Unpleasant Word Stimuli Concerning Body Image: An fMRI Study,” British Journal of Psychiatry, Vol. 186, 2005, pp. 48-53. doi:10.1192/bjp.186.1.48

[5] H. M. Mohr, J. Zimmermann, C. Röder, C. Lenz, G. Overbeck and R. Grabhorn, "Separating Two Components of Body Image in Anorexia Nervosa Using fMRI," Psychological Medicine, Vol. 40, 2010, pp. 1519-1529. doi:10.1017/S0033291709991826

[6] H. C. Friederich, R. Uher, S. Brooks, V. Giampietro, M. Brammer, S. C. Williams, W. Herzog, J. Treasure and I. C. Campbell, "I'm Not as Slim as That Girl: Neural Bases of Body Shape Self-Comparison to Media Images," NeuroImage, Vol. 37, No. 2, 2007, pp. 674-681. doi:10.1016/j.neuroimage.2007.05.039

[7] M. Kurosaki, N. Shirao, H. Yamashita, Y. Okamoto and S. Yamawaki, "Distorted Images of One's Own Body Activates the Prefrontal Cortex and Limbic/Paralimbic System in Young Women: A Functional Magnetic Resonance Imaging Study,” Biological Psychiatry, Vol. 59, No. 4, 2006, pp. 380-386. doi:10.1016/j.biopsych.2005.06.039

[8] P. Fossati, S. J. Hevenor, S. J. Graham, C. Grady, M. L. Keightley, F. Craik and H. Mayberg, "In Search of the Emotional Self: An FMRI Study Using Positive and Negative Emotional Words," American Journal of Psychiatry, Vol. 160, No. 11, 2003, pp. 1938-1945. doi:10.1176/appi.ajp.160.11.1938

[9] K. N. Ochsner, K. Knierim, D. H. Ludlow, J. Hanelin, T. Ramachandran, G. Glover and S. C. Mackey, "Reflecting upon Feelings: An fMRI Study of Neural Systems Supporting the Attribution of Emotion to Self and Other," Journal of Cognitive Neuroscience, Vol. 16, No. 10, 2004, pp. 1746-1772. doi:10.1162/0898929042947829

[10] P. Sachdev, N. Mondraty, W. Wen and K. Guilford, "Brains of Anorexia Nervosa Patients Process Self-Images Differently from Non-Self-Images: An fMRI Study,” Nueropsyhologia, Vol. 46, 2008, pp. 2161-2168.

[11] D. A. Boas, A. M. Dale and M. A. Franceschini, "Diffuse Optical Imaging of Brain Activation: Approaches to Optimizing Image Sensitivity, Resolution, and Accuracy,” Neuroimage, Vol. 23, Supplement 1, 2004, pp. 275-288. doi:10.1016/j.neuroimage.2004.07.011

[12] M. Suda, M. Fukuda, T. Sato, S. Iwata, M. Song, M.
Kameyama and M. Mikuni, "Subjective Feeling of Psychological Fatigue Is Related to Decreased Reactivity in Ventrolateral Prefrontal Cortex,” Brain Research, Vol. 1252, Supplement 1, 2009, pp. 152-160.

doi:10.1016/j.brainres.2008.11.077

[13] S. Grignon, K. Forget, M. Durand and T. Huppert, "Increased Left Prefrontal Activation During Staring/Mutism Episodes in a Patient with Resistant Catatonic Schizophrenia: A Near Infrared Spectroscopy Study,” Cognitive Behavior Neurology, Vol. 21, No. 1, 2008, pp. 41-45. doi:10.1097/WNN.0b013e3181684d87

[14] R. Takizawa, K.Kasai, Y. Kawakubo, K. Marumo, S. Kawasaki and H. Yamasue, "Reduced Frontopolar Activation during Verbal Fluency Task in Schizophrenia: A Multichannel Near-Infrared Spectroscopy Study,” Schizophrenia Research, Vol. 99, 2008, pp. 250-262. doi:10.1016/j.schres.2007.10.025

[15] K. Matsuo, Y. Onodera, T. Hamamoto, K. Muraki, N. Kato and T. Kato, "Hypofrontality and Microvascular Dysregulation in Remitted Late-Onset Depression Assessed by Functional Near-Infrared Spectroscopy,” Neuroimage, Vol. 26, No. 1, 2005, pp. 234-242. doi:10.1016/j.neuroimage.2005.01.024

[16] A. C. Ehlis, C. G. Bähne, C. P. Jacob, M. J. Herrmann and A. J. Fallgatter, "Reduced Lateral Prefrontal Activation in Adult Patients with Attention-Deficit/Hyperactivity Disorder (ADHD) during a Working Memory Task: A Functional Near-Infrared Spectroscopy (fNIRS) Study,” Journal of Psychiatric Research, Vol. 42, No. 3, 2008, pp. 1060-1067. doi:10.1016/j.jpsychires.2007.11.011

[17] M. Suda, Y. Takei, Y. Aoyama, K. Narita, T. Sato, M. Fukuda and M. Mikuni, "Frontopolar Activation During Face-to-Face Conversation: An in Situ Study Using Near-Infrared Spectroscopy,” Neuropsychologia, Vol. 48, No. 2, 2010a, pp. 441-447. doi:10.1016/j.neuropsychologia.2009.09.036

[18] M. Suda, T. Sato, M. Kameyama, M. Ito, T. Suto, Y. Yamagishi, T. Uehara, M. Fukuda and M. Mikuni, "Decreased Cortical Reactivity Underlies Subjective Daytime Light Sleepiness in Healthy Subjects: A Multichannel Near-Infrared Spectroscopy Study," Neuroscience Research, Vol. 60, No. 3, 2008, pp. 319-326. doi:10.1016/j.neures.2007.12.001

[19] T. Sato, M. Ito, T. Suto, M. Kameyama, M. Suda, Y. Yamagishi, A. Ohshima, T. Uehara, M. Fukuda and M. Mikuni, "Time Courses of Brain Activation and Their Implications for Function: A Multichannel Near-Infrared Spectroscopy Study during Finger Tapping," Neuroscience Research, Vol. 58, No. 3, 2007, pp. 297-304. doi:10.1016/j.neures.2007.03.014

[20] E. Ohmae, Y. Ouchi, M. Oda, T. Suzuki, S. Nobesawa, T. Kanno, E. Yoshikawa, M. Futatsubashi, Y. Ueda, H. Okada and Y. Yamashita, "Cerebral Hemodynamics Evaluation by Near-Infrared Time-Resolved Spectroscopy: Correlation with Simultaneous Positron Emission Tomography Measurements,” Neuroimage, Vol. 29, No. 3, 2006, pp. 697705.

[21] V. Toronov, A. Webb, J. H. Choi, M. Wolf, A. Michalos, E. Gratton and D. Hueber, "Investigation of Human Brain 
Hemodynamics by Simultaneous Near-Infrared Spectroscopy and Functional Magnetic Resonance Imaging,” Medical Physiology, Vol. 28, No. 4, 2001, pp. 521-527. doi:10.1118/1.1354627

[22] D. J. Mehagnoul-Schipper, B. F. van der Kallen, W. N. Colier, M. C. van der Sluijs, L. J. van Erning, H. O. Thijssen, B. Oeseburg, W. H. Hoefnagels and R. W. Jansen, "Simultaneous Measurements of Cerebral Oxygenation Changes During Brain Activation by Near-Infrared Spectroscopy and Functional Magnetic Resonance Imaging in Healthy Young and Elderly Subjects," Human Brain Mapping, Vol. 16, No. 1, 2002, pp. 14-23. doi:10.1002/hbm.10026

[23] D. M. Garner, M. P. Olmsted, Y. Bohr and P. E. Garfinkel, "The Eating Attitudes Test: Psychometric Features and Clinical Correlates," Psychological Medicine, Vol. 12, No. 4, 1982, pp. 871-878. doi:10.1017/S0033291700049163

[24] K. Nakamura, Y. Hoshino, A. Watanabe, K. Honda, S. Niwa, K. Tominaga, S. Shimai and M. Yamamoto, "Eating Problems in Female Japanese High School Students: A Prevalence Study," International Journal of Eating Disorders, Vol. 26, No. 1, 1999, pp. 91-95. doi:10.1002/(SICI)1098-108X(199907)26:1<91::AID-EA T12>3.0.CO;2-M

[25] M. Kameyama, M. Fukuda, Y. Yamagishi, T. Sato, T. Uehara, M. Ito and M. Mikuni, "Frontal Lobe Function in Bipolar Disorder: A Multichannel Near-Infrared Spectroscopy Study," Neuroimage, Vol. 29, No. 1, 2006, pp. 172-184. doi:10.1016/j.neuroimage.2005.07.025

[26] T. Suto, M. Fukuda, M. Ito, T. Uehara and M. Mikuni, "Multichannel Near-Infrared Spectroscopy in Depression and Schizophrenia: Cognitive Brain Activation Study,” Biological Psychiatry, Vol. 55, No. 5, 2005, pp. 501-511. doi:10.1016/j.biopsych.2003.09.008

[27] A. Etkin, T. Egner, D. M. Peraza, E. R. Kandel and J. Hirsch, "Resolving Emotional Conflict: A Role for the Rostral Anterior Cingulate Cortex in Modulating Activity in the Amygdale," Neuron, Vol. 51, No. 6, 2006, pp. 871882. doi:10.1016/j.neuron.2006.07.029

[28] M. Suda, T. Uehara, M. Fukuda, T. Sato, M. Kameyama, and M. Mikuni, "Dieting Tendency and Eating Behavior Problems in Eating Disorder Correlate with Right Frontotemporal and Left Orbitofrontal Cortex: A Near-Infrared Spectroscopy Study,” Journal of Psychiatry Research, Vol. 44, No. 8, 2010b, pp. 547-555. doi:10.1016/j.jpsychires.2009.11.005

[29] T. A. Kimbrell, M. S. George, P. I. Parekh, T. A. Ketter, D. M. Podell, A. L. Danielson, J. D. Repella, B. E. Benson, M. W. Willis, P. Herscovitch and R. M. Post, "Regional Brain Activity During Transient Selfinduced Anxiety and Anger in Healthy Adults,” Biological Psychiatry, Vol. 46, No. 4, 1999, pp. 454-465. doi:10.1016/S0006-3223(99)00103-1

[30] D. D. Dougherty, L. M. Shin, N. M. Alpert, R. K. Pitman, S. P. Orr, M. Lasko, M. L. Macklin, A. J. Fischman, S. L. Rauch, "Anger in Healthy Men: A Pet Study Using Script-Driven Imagery,” Biological Psychiatry, Vol. 46, No. 4, 1999, pp. 466-472. doi:10.1016/S0006-3223(99)00063-3

[31] S. Grimm, C. F. Schmidt, F. Bermpohl, A. Heinzel, Y. Dahlem, M. Wyss, D. Hell, P. Boesiger, H. Boeker and G. Northoff, "Segregated Neural Representation of Distinct Emotion Dimensions in the Prefrontal Cortex-An fMRI Study,” Neuroimage, Vol. 30, No. 1, 2006, pp. 325-340. doi:10.1016/j.neuroimage.2005.09.006

[32] D. Tsuzuki, V. Jurcak, A. K. Singh, M. Okamoto, E. Watanabe and I. Dan, "Virtual Spatial Registration of StandAlone fNIRS Data to MNI Space,” Neuroimage, Vol. 34, No. 4, 2007, pp. 1506-1518. doi:10.1016/j.neuroimage.2006.10.043

[33] P. E. Downing, Y. Jiang, M. Shuman and N. Kanwisher, "A Cortical Area Selective for Visual Processing of the Human Body,” Science, Vol. 293, No. 5539, 2001, pp. 2470-2473. doi:10.1126/science.1063414

[34] D. Nico, E. Daprati, N. Nighoghossian, E. Carrier, J. R. Duhamel and A. Sirigu, "The Role of the Right Parietal Lobe in Anorexia Nervosa,” Psychological Medicine, Vol. 40, No. 9, 2010, pp. 1531-1539. doi:10.1017/S0033291709991851

[35] Y. Miyake, Y. Okamoto, K. Onoda, M. Kurosaki, N. Shirao, Y. Okamoto and S. Yamawaki, "Brain Activation during the Perception of Distorted Body Images in Eating Disorders,” Psychiatry Research, Vol. 181, No. 3, 2010a, pp. 183-192. doi:10.1016/j.pscychresns.2009.09.001

[36] Y. Miyake, Y. Okamoto, K. Onoda, N. Shirao, Y. Okamoto, Y. Otagaki and S. Yamawaki, "Neural Processing of Negative Word Stimuli Concerning Body Image in Patients with Eating Disorders: An fMRI Study,” Neuroimage, Vol. 50, No. 3, 2010b, pp. 1333-1339. doi:10.1016/j.neuroimage.2009.12.095 